\title{
SPACE DENSITIES FOR POWERFUL RADIO SOURCES IN THE LIGHT OF UNIFICATION
}

\author{
C.A. JACKSON \\ Institute of Astronomy, University of Cambridge, \\ Cambridge, CB3 OHA, UK \\ AND \\ J.V. WALL \\ Royal Greenwich Observatory, Madingley Road, \\ Cambridge, CB3 OEZ, UK
}

As radio survey frequency is raised the proportion of flat-spectrum sources increases in bright flux-limited samples ( $\mathrm{eg}$ Wall 1994, Aust $J$ Phys 47, 625). Differential source counts show a corresponding broadening of the central maximum due to the increasing proportion of flat-spectrum sources. Orr \& Browne (1982, MNRAS 200, 1067) modelled this change in shape of the source count by proposing a unifying scheme which states that the core-dominated, flat-spectrum radio sources are the steep-spectrum sources with their cores Doppler-boosted due to the alignment of the jets with the line of sight.

Investigation of the space densities of radio sources should proceed with populations which are physically delineated; in the face of unified models, the traditional division into 'flat-spectrum' and 'steep-spectrum' populations is incorrect. To this end we are undertaking a new space-density analysis to explore the implications of unified-model schemes, including both the radio-loud QSO - FRII radio-galaxy paradigm and the BL Lac - FRI radio-galaxy paradigm (see Urry and Padovani 1995, PASP 107, 803). To test the formalism, our first stage described here uses (1) complete samples and source-count data over a wide frequency range and (2) optimizing techniques to explore parameterized evolution and beaming models.

This initial analysis followed the scheme developed by Wall et. al (1980, $M N R A S 193,683)$. Together with a $151-\mathrm{MHz}$ source-count, the 162 steepspectrum sources in the 3 CR sample (Laing et. al 1983, MNRAS 204, 151) were used to define the epoch-dependent luminosity function of the 'parent' 
population. The best-fit parameters were determined using the AMOEBA downhill simplex method in multidimensions (Press et. al 1992, Numerical Recipes in Fortran (CUP), 402), evaluating $\chi^{2}$ between the observed and model source counts. For evolution of the form $\exp \left(M\left(1-t / t_{0}\right)\right)$ the optimal parameters $(\Omega=1, h=0.5)$ are $M=10.92, z_{c}=4.075$ and transition powers between evolving and non-evolving sources at $\log _{10}\left(P_{1}\right)=25.33$, $\log _{10}\left(P_{2}\right)=27.57$. This demonstrates that modern data comprising complete redshifts for the $3 \mathrm{CR}$ sources plus a deep source count require a redshift cut-off in the space density for steep-spectrum sources.

These parameter values and a single spectral index of -0.75 were used to estimate the $5 \mathrm{GHz}$ count of steep-spectrum sources (Figure 1). Inclusion of the flat-spectrum, beamed population at $5 \mathrm{GHz}$ was achieved with two additional parameters, the Lorentz factor $\gamma$ and the rest frame core-toextended flux ratio $R_{c}$. The observed core-to-extended flux ratio $R_{o b s}$ is given by $R_{o b s}=R_{c}\left([\gamma(1-\beta \cos \theta)]^{-2+\alpha_{f l a t}}+[\gamma(1+\beta \cos \theta)]^{-2+\alpha_{f l a t}}\right)$ for a source comprising a pair of continuous relativistic jets with bulk plasma velocity $\beta c$ whose ejection axis is aligned at a random angle $\theta\left(\geq 0^{\circ}, \leq 90^{\circ}\right)$ to the line of sight. We adopted $\alpha_{\text {flat }}=0.0$, and took a source as being 'flat-spectrum' for small enough values of $\theta<\theta_{c}$ such that $R_{o b s} \geq 1.0$ and its observed flux density $S_{\text {enhanced }}=R_{o b s} . S_{\nu_{1}}$. For $\gamma=10.0$ and $R_{c}=$ $0.02\left(\theta_{c}=8^{\circ}\right)$, the count of flat-spectrum sources summed with the steepspectrum source count closely follows the observed count (Figure 1).

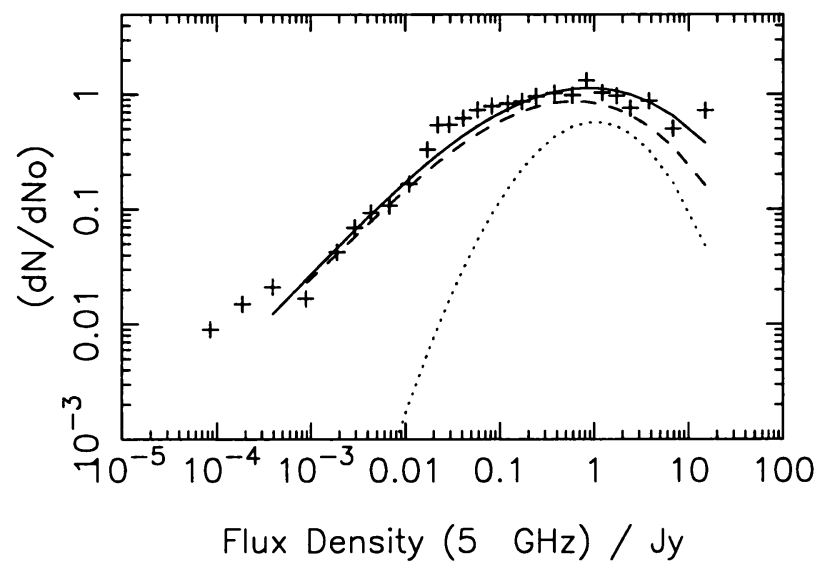

Figure 1. Model and observed source counts at $5 \mathrm{GHz}:++++$ observed source count, - - - model count for steep-spectrum objects, ... model count for flat-spectrum objects, — total model source count.

This initial analysis demonstrates that (a) a diminution in the space density of 'parent' sources at redshifts above 4 is required, and (b) the FRII - radio-loud QSO unified scheme is consistent with the high-frequency count data for reasonable beaming parameters. 